\title{
High Speed Hybrid Object Tracking Algorithm using Image and Event Data
}

This paper was downloaded from TechRxiv (https://www.techrxiv.org).

\section{LICENSE}

CC BY 4.0

SUBMISSION DATE / POSTED DATE

03-11-2021 / 05-11-2021

\section{CITATION}

El Shair, Zaid; Rawashdeh, Samir A. (2021): High Speed Hybrid Object Tracking Algorithm using Image and Event Data. TechRxiv. Preprint. https://doi.org/10.36227/techrxiv.16926610.v1

$\mathrm{DOI}$

10.36227/techrxiv.16926610.v1 


\title{
High-speed Hybrid Object Tracking Algorithm using Image and Event Data
}

\author{
Zaid A. El Shair, Samir A. Rawashdeh, Senior Member, IEEE
}

\begin{abstract}
Event cameras are novel, bio-inspired, imagers that output per-pixel brightness changes at $\sim 1 \mu$ s latency. Besides their high response rates, they offer numerous advantages over conventional cameras, such as no motion blur, high dynamic range, and low power consumption. However, event cameras suffer from some limitations such as lacking the intensity information that regular cameras provide. In this paper, we present a hybrid object tracking algorithm that leverages both images and events, thus, providing a complementary approach that utilizes some of the advantages of both imaging types. Our tracking algorithm detects the objects in the image frames, then tracks objects in the blind time between consecutive frames using per-object event masks extracted from the event data. Moreover, we set up a data collection experiment to evaluate and analyze our algorithm's performance using Dynamic and Active-Pixel Vision Sensor (DAVIS), which combines a monochrome camera as well as an event-based sensor using the same pixel array. Results show that our tracking algorithm can reach up to $500 \mathrm{~Hz}$ tracking rates based on a standard image framerate of $24 \mathrm{~Hz}$ and asynchronous event-data data collected by the hybrid camera.
\end{abstract}

Index Terms-Computer Vision, Dynamic Vision Sensors, Event Cameras, Hybrid Vision, Multi-Sensor Tracking, Object Detection, Object Tracking.

\section{Introduction}

$\mathrm{F}$ OR decades, computer vision has heavily relied on typical intensity images that are captured by conventional cameras at fixed rates. The research advancements of computer vision throughout the last half-century have created numerous applications in this domain, based on the output of these conventional cameras. Image-processing-based tasks such as object detection [1,2], classification $[3,4]$, and segmentation [5] are some of the basic and vital components that play a role in perception and decision making in robotics in general. All of which enable different applications such as object recognition and tracking [6], video surveillance [7], and much more.

There are several physical limitations of conventional frame-based cameras that constrain their robustness and

This paragraph of the first footnote will contain the date on which you submitted your paper for review. It will also contain support information, including sponsor and financial support acknowledgment. For example, "This work was supported in part by the U.S. Department of Commerce under Grant BS123456."

The next few paragraphs should contain the authors' current affiliations, including current address and e-mail. For example, F. A. Author is with the National Institute of Standards and Technology, Boulder, CO 80305 USA (e-mail: author@ boulder.nist.gov).

S. B. Author, Jr., was with Rice University, Houston, TX 77005 USA. $\mathrm{He}$ is now with the Department of Physics, Colorado State University, Fort Collins, CO 80523 USA (e-mail: author@lamar.colostate.edu).

T. C. Author is with the Electrical Engineering Department, University of Colorado, Boulder, CO 80309 USA, on leave from the National Research Institute for Metals, Tsukuba, Japan (e-mail: author@nrim.go.jp). reliability for different timing and safety-critical applications [8], such as autonomous driving and high-speed robotics. These limitations include low dynamic range, motion blur, high-power consumption, and relatively low update rates. Some of these can be mitigated, such as the low update rate limitation, however, at the cost of higher power consumption due to greater computational power requirements. This is mainly due to the significantly higher data output that mostly consists of redundant data. On the other hand, Dynamic Vision Sensors (DVS) do not suffer from these limitations [9].

DVS, also known as Event cameras, are novel, bio-inspired, imaging devices that were first introduced in 2008 by Lichtsteiner et. al [10]. Unlike standard cameras, event cameras can generate asynchronous per-pixel output, known as events. An event can be defined as a pixel that increases or decreases in brightness anytime the $\log$ of the magnitude of brightness change is higher than a set threshold. Each event $e_{i}$ reported contains 4 different parameters: $\mathrm{x}$ and $\mathrm{y}$ pixel coordinate at which they have appeared, a microsecond timestamp $t_{i}$, and a binary polarity $p_{i}$ which can be either +1 or -1 , indicating a positive or a negative brightness change, respectively. Event cameras offer numerous advantages (compared to standard cameras) including high dynamic range (HDR) of $\sim 120 \mathrm{~dB}$ vs $60 \mathrm{~dB}$, low latency ( 1 $\mu$ s per event), and low power consumption.

Event cameras have introduced many applications such as low-power monitoring and surveillance [11], high-speed obstacle detection and avoidance [12,13], the ability to deblur blurry videos [14], and advanced Simultaneous Localization 
and Mapping (SLAM) that utilizes images, inertial data, and events, enabling SLAM in high-speed and HDR scenarios. These are a few of many possible applications of event cameras enabled by their very novel nature, creating a potential for new research in multiple domains.

However, one of the main disadvantages of Event cameras is the lack of intensity information (an event reports only a binary polarity brightness change without magnitude) causing various computer vision tasks, such as object detection and classification, to be quite challenging.

Gehrig et. al [15], argues that event data, on its own, should be sufficient to design efficient algorithms that perform all computer vision tasks; as the stream of events should, in theory, contain all the visual information through any arbitrary period of time. However, the design of these end-to-end learning algorithms is not yet fully possible due to the lack of ample amounts of data for training. Instead, it is possible to reconstruct intensity images at a high frame rate, for which, common computer vision algorithms (typically designed for conventional cameras) yield state-of-the-art performance with the event-data-based reconstructed images [16]. On the other hand, a pure event-based approach by using reconstructed frames might not always be ideal due to the noisy nature of event cameras in real life, resulting in some artifacts and possibly unrealistic reconstructions, besides the high computation requirements. Moreover, image reconstruction from events is a bit problematic when using a stationary camera.

One of the contemporary solutions to the aforementioned constraints of both frame-based and event-based computer vision tasks is to utilize both approaches within one. Such solutions (referred to as a hybrid approach) would combine the advantages of both imaging devices. We present a high-speed hybrid object tracking algorithm that utilizes both high-rate asynchronous event data, as well as synchronous image frames, to enable very high object tracking rates. This work is done using a Dynamic and Active-Pixel Vision Sensor (DAVIS) 240c camera, that includes both a frame-based monochrome camera and a DVS within the same camera lens, at a resolution of $240 \times 180$ pixels. Furthermore, we built our solution on a traffic monitoring dataset collected using a stationary DAVIS 240c. We focus on some of the advantages of these event cameras in the domain of smart infrastructure and smart traffic lights in general as some possible applications, but mainly for high-speed continuous object detection and tracking specifically. Moreover, we test our hybrid algorithm by conducting a data collection experiment to quantify the performance of our tracking algorithm.

\section{RELATED WORK}

In the literature, event-based tracking, in general, has been mainly feature-based. Most of the work focuses on the event data as a means to extract either features or corners to track. Event-based tracking enables the possibility of inter-frame tracking, i.e., tracking between the frames of a standard camera. As in any tracking application, it requires data association of what is being tracked across time, which is a challenge for event data, as they vary significantly in nature compared to typical images, such as reporting only per-pixel brightness changes and dependency on motion to generate data in a static scene. Early adaptations of feature-based tracking heavily relied on event-based clustering to track objects across the scene [17].

Different approaches exist for tracking using an event camera. One approach of feature tracking is using a hybrid between a frame-based monochrome camera and an events camera (using DAVIS) by David Tedaldi et al. [18] which is based on finding all the corners in the image using a Harris detector then running a canny edge detector on this image to create a binary map. Finally, extract local edge-map patch around corner points to track the features. Another method developed and evaluated by Gehrig et. al, similarly, uses spatial gradient on the image frame to extract features and generate a template that is used to subsequently track these features asynchronously using events, based on a generative event model [19]. Moreover, they argue that the frames from conventional cameras, in their method, can be replaced with frames purely reconstructed from events, eliminating the need of having a hybrid camera (a standard intensity camera and an event camera) whereas an event camera would be sufficient, albeit with higher computational costs.

In contrast, event-based recognition algorithms have had numerous advancements lately, such as performing recognition on different types of objects [20, 21]. However, the main challenge of performing event-based object recognition is the dependency on motion to generate events, causing the event-based approach of detecting still or slowmoving objects to be problematic, and more complicated for moving objects due to the added temporal component.

Initial object detection and tracking results of a dataset collected using a fixed camera have shown worse performance when using reconstructed frames generated from event data, even when compared to standard frames only. This is mainly due to the low resolution of the DAVIS camera and the significantly low number of events generated when the imaging device itself is held static without any motion. This is mainly caused by the fact that events are only generated around the edges of the objects when there's a change in the scene, where a fixed camera would not generate any events relating to the static background of the scene.

On the other hand, object tracking using conventional camera frames has been vast and well-established for quite some time [22,23]. Typically, object trackers utilize an object detection mechanism, performed on image frames, to detect and track all the objects across sequential frames, based on some metric. This results in discrete tracking data, even when considering $100 \%$ successful object detection, which might not be sufficient for high-speed robotics or if a higher temporal resolution is required based on the application in mind. Most common regular cameras capture images at a relatively low rate of about $30 \mathrm{~Hz}$ (or frames per second). Therefore, a rapid change in the position of the object being tracked might not be detected if it occurs at a higher rate than the camera's frame capture rate. The effects of this might not 
be drastic based on the intended application, however, tracking ends up yielding very low temporal resolution results with insufficient data to infer other useful information such as object acceleration or deceleration rates, velocity, or having continuous tracking results overall. Frame-based object detection and tracking suffer from other drawbacks, such as not being able to detect objects in some of the frames, resulting in even more discontinuous tracking results, where the best tracking rate possible would be to match the camera's frame capture rate.

In our work, we utilize both image and event data to track objects across the scene using our Hybrid Object Tracking algorithm. Moreover, we constrain the problem of vehicle tracking as our approach has been mainly developed to track vehicles due to their fixed-shaped edges with limited dynamic components. We utilize deep-learning-based object detectors for frame-based object detection and localization while utilizing event data for a computationally inexpensive interframe object tracking that is based on sparse matrix multiplication. Event masks, used for inter-frame tracking, are extracted using the frame-based object's bounding box making it robust to shadows that can be misclassified using event data only, and is robust to noise as, unlike event-based approaches, not all events are classified or utilized in tracking. Only events within the regions of interest are considered. Finally, our approach unlocks the capabilities of tracking objects at over 510 times the original tracking rate of the frame-based only approach, yielding tracking rates as high as $500 \mathrm{~Hz}$ testing the limits of this hybrid approach. Moreover, a data collection experiment is presented to evaluate our approach.

\section{HyBrid OBJect TRACKING Algorithm}

In this section, the main contribution of this paper, which is our hybrid object tracking, is introduced. Our algorithm relies on data from both the regular camera (image frames) and the DVS's event data, therefore, referred to as a hybrid algorithm.

A vital first step for tracking objects across time is to detect them when they first appear and in every subsequent frame. As mentioned before, classification using event data alone is challenging, therefore, our algorithm uses the image frames to detect and classify objects wherever they appear in the scene, then tracks them between frames using event data. In this work, we utilize some of the common, pre-trained, deeplearning-based object detectors, specifically YOLOv3 [24] and SSD [25]. These models are used to detect objects in every new image frame as shown in Fig. 1. The frame-based object detectors can be replaced by other frame-based detectors as needed. Below, we break down the utilized tracking algorithm, before going in-depth on our hybrid tracking algorithm's design. Note that our work, for now, does not focus on delivering real-time performance, however, it can be optimized and compiled properly to be able to do so.

\section{A. Centroid tracking algorithm}

As for the object tracker, we utilize a centroid-based (detections' center $\mathrm{x}$ and $\mathrm{y}$ coordinates) tracking algorithm based on Euclidean Distance [26], which is a common metric

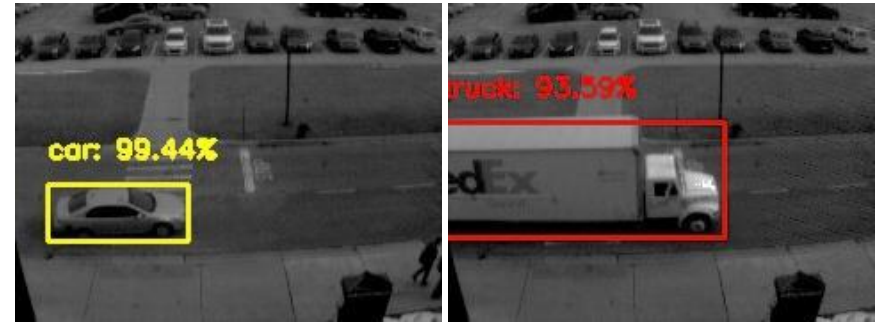

Fig. 1. Object detection on sample images using YOLOv3 Object Detector. In this scene, static objects like the parked vehicles in the top half of the scene are ignored.

that is used to successfully track objects across subsequent frames. This metric is appropriate for our use case due to the continuous nature of the event data and, thus, the resulting tracking data. Moreover, the centroid tracking algorithm used is based on Adrian Rosebrock's implementation [27]. The centroid tracking algorithm is summarized as follows:

A.Detect Objects in the frame:

1. Using an object detector, detect all the objects in the initial frame.

2. Compute the centroid of each.

3. Assign unique IDs for each of the detected objects.

B.Compute Euclidean distance between new bounding boxes and existing objects:

1. Detect objects in every subsequent frame.

2. Compute Euclidean distance between each pair of existing centroids and newly detected centroids.

C. Update the centroid coordinates of existing objects:

1. Update existing objects' centroids according to the shortest distances with the new detections.

D.Register new objects, and deregister old objects:

1. Associate any new and unmatched objects with unique IDs and store their positions.

2. Deregister any unmatched existing objects after $\mathrm{N}$ subsequent unmatched frames.

\section{B. Hybrid Object Tracking Algorithm using Standard Image Frames and Event data}

Initially, the window frame size is set. The window frame size refers to the size of the time period that the algorithm will read and accumulate data throughout (both image and event

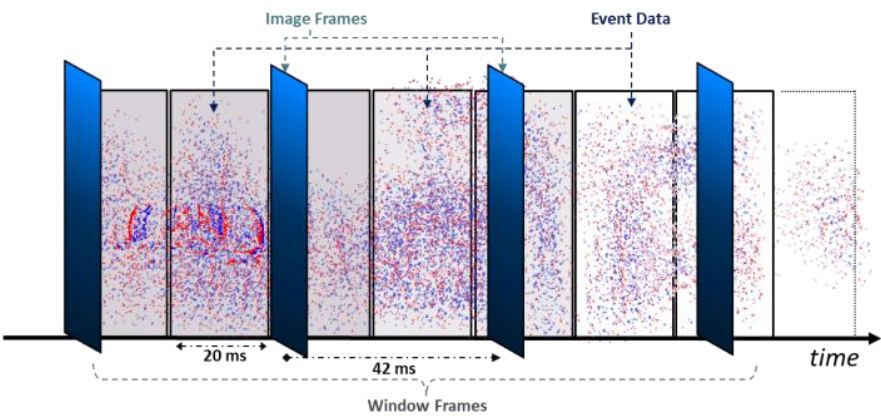

Fig. 2. Visualization of synchronized stream of image frames and event data over time. In this example, the image frames are captured every $42 \mathrm{~ms}$ (at a rate of $24 \mathrm{~Hz}$ ), whereas the window frame size is set to $20 \mathrm{~ms}$ (tracking rate of $50 \mathrm{~Hz}$ ). A window frame encapsulates any image frames and event data available in that specified time frame. 


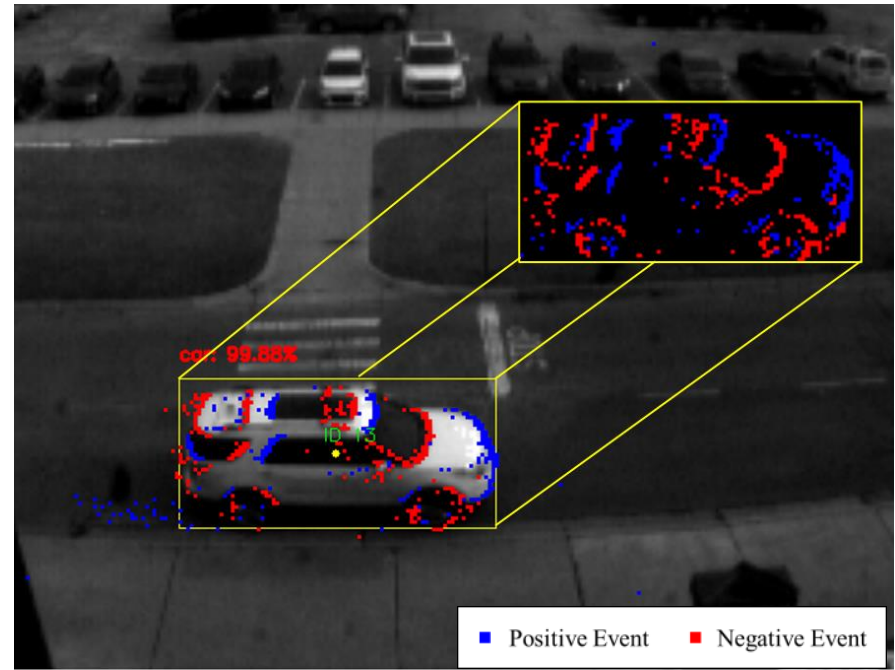

Fig. 3. Successful Object Detection of a White SUV using the grayscale image frame data shown in the yellow bounding box.

data), which defines the tracking rate. For example, if it is set to $20 \mathrm{~ms}$, then the window will read $20 \mathrm{~ms}$ of the available synchronized event data and image frames (whenever available).

DAVIS

240c used has a frame-based capture rate of $24 \mathrm{~Hz}$, therefore, a new image frame is read around every $42 \mathrm{~ms}$. Accordingly, at this window frame size of $20 \mathrm{~ms}$, a new image frame is loaded around every 2 window frames. As a result, the other window frames would contain only event data, as visualized in Fig. 2. A window frame size of $20 \mathrm{~ms}$ yields a $50 \mathrm{~Hz}$ tracking rate. Similarly, window frame sizes of 10,5 , and 2 milliseconds yield 100, 200, and $500 \mathrm{~Hz}$ tracking rates, respectively.

Based on the chosen window frame size, the hybrid algorithm is designed to operate as follows:

- Step 1 - Load a new window frame, typically containing both an image frame and accumulated event data.

- Step 2 - Detect Objects in the image frame captured by the monochrome camera.

- Step 3 - If no objects were detected, repeat from step 1. Otherwise, if an object was detected (similar to Fig. 3):

1. Update the centroid tracking algorithm with the detected object's centroid coordinates to register a new object.

2. Create an event mask for each of the detected objects by extracting all of the accumulated events in the last window frame within the bounding box detection in the image frame (an example event mask extracted is

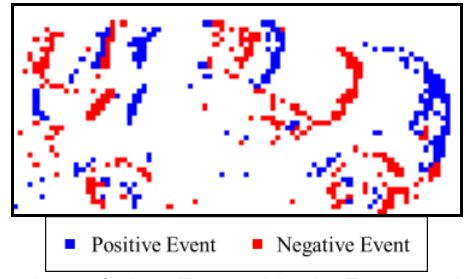

Fig. 4. Visualization of the Event Mask Extracted of the detected White SUV shown in Fig. 3. This mask is stored in the form of a sparse matrix. shown in Fig. 4). Positive and negative events are stored as +1 and -1 integers, respectively.

3. Load the next window frame that contains the synchronized image frames and event data available within the set window frame size.

- Step 4 - when loading a new window frame that contains only event data:

a) Create a search region centered around the centroids of each of the 's currently being tracked. The search region used is $10 \%$ larger than the framebased detection's bounding box dimensions in each direction. Thus, around 44\% larger bounding box size in our case (represented by the green bounding box in Fig. 5).

b) Extract all the new events (available in the current window frame) residing within the search region's area and create a sparse matrix of these events.

c) Create a sparse matrix of the event mask extracted earlier in step 2(b), and a subset of the search region.

d) Perform cross-correlation between the mask and search region as demonstrated in Fig. 6. This process is mainly a 2-dimensional matrix multiplication between the event mask and the subsection of the search region, starting at the top left corner of the search region, then calculating the sum of all the cells in the resulting matrix and storing the result in a separate cost matrix.

e) Use the highest correlating box as the object's new updated center position. Fig. 5 shows the best tracking result of this maximum correlation step highlighted in the cyan bounding box, which is the best fit event-based tracking for the current window frame. A minimum threshold is typically applied so that the system will only update each

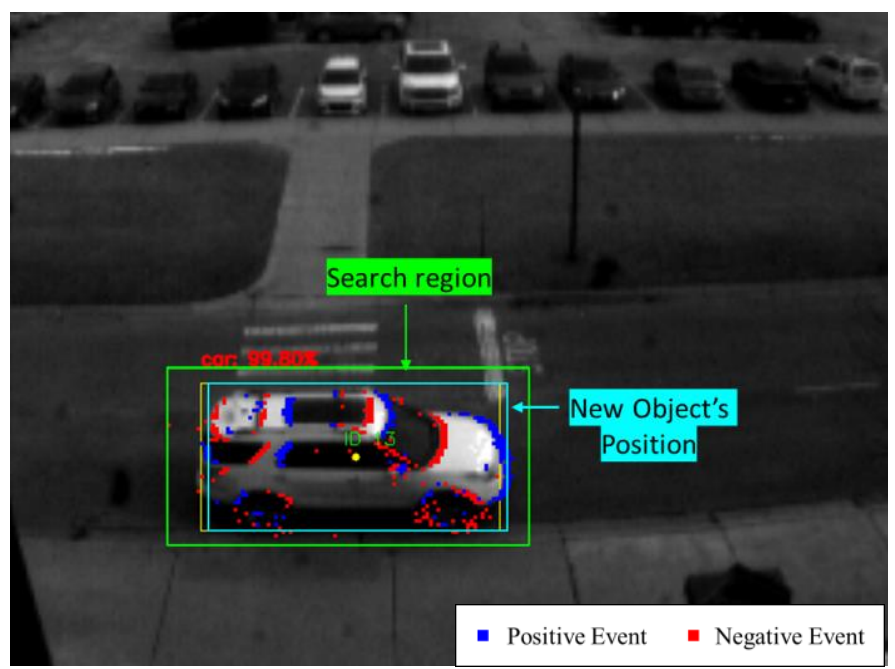

Fig. 5. White SUV being successfully tracked at $\sim 60 \mathrm{~Hz}$ using Frame and Event data. The Yellow box represents the initial object detection of the last camera image frame, whereas the green bounding box demonstrates the search region for the latest window frame, while the cyan box demonstrates the successful tracking of the events between frames. 


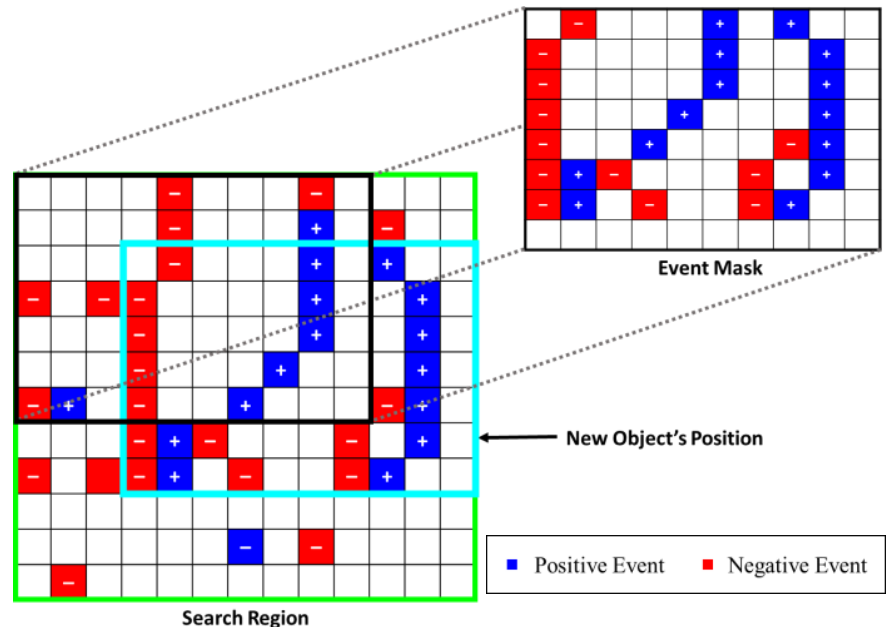

Fig. 6. Sparse Matrix local multiplication of the event mask and a sliding section of the search region, to find the highest correlating position of the object by summing the result of each multiplication, similar to a typical image convolution using a kernel. After completing the cross-correlation the new object's location is established by selecting the highest correlating position in the search region of the new window frame as is highlighted in the cyan bounding box.

object's position if the highest correlating position is above a set threshold. This is typically done to avoid updating the object's position based on some noise and adds some robustness to the tracking.

f) If successfully tracked, update the object's position using the centroid tracker, then update its event mask by aggregating it with the new event data available within the most recent updated position (assuming that the new events line up correctly with the previous events). This step typically adds robustness to the system, particularly when tracking at very high rates, such as over $200 \mathrm{~Hz}$, where fewer events are generated.

- Step 5 - load a new window frame and repeat from step 4 to track the objects between frames by only using the incoming event data until a window frame containing an image is read. At that point, repeat from step 1.

During step 4, we employ sparse matrices to perform a more efficient inter-frame object tracking using event data to find the highest correlating position. Sparse matrices are suitable since event data is inherently sparse, as the high majority of pixels would not hold any events.

Overall, our hybrid tracking algorithm is summarized and demonstrated in a flowchart as shown in Fig. 7 for a more streamlined visual representation.

\section{DATA COLlection}

In order to analyze and quantify the performance of the aforementioned hybrid tracking algorithm, some ground truth data is required. However, as discussed earlier, annotated ground truth data for event data sequences remains to be quite a challenge. The data collected is used to test the accuracy of our tracking algorithm that is produced at high rates and

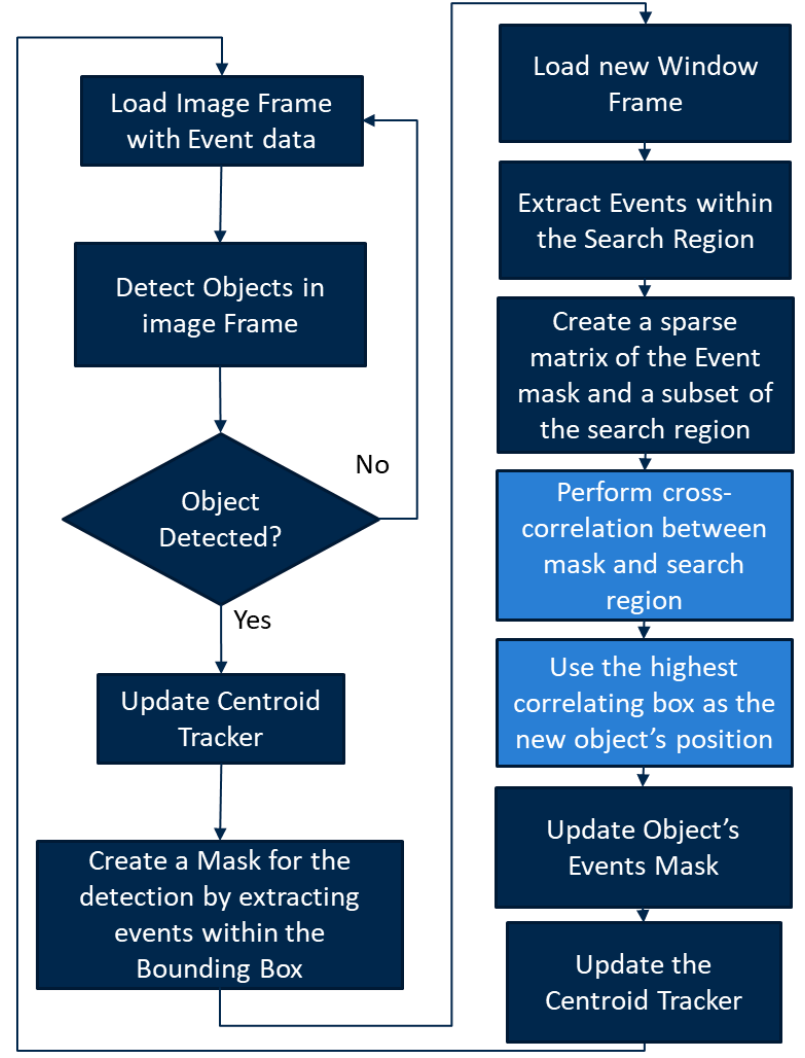

Fig. 7. Flowchart of the Hybrid Object Tracking Algorithm.

evaluate it both qualitatively and quantitatively. Analyzing and testing tracking algorithms designed to run at very high rates is quite challenging, as typical position estimation devices (such as GPS or accelerometers) would yield relatively low update rates $(\sim 10 \mathrm{~Hz})$ that are significantly lower than the update rates this tracking algorithm is targeting (100$1000 \mathrm{~Hz})$.

Initially, a simple dataset was collected using DAVIS 240c, which was set up on a top floor of a building facing downwards at an angle with a street with a stop sign and crosswalk, to imitate a bird's eye view. This dataset was mainly used to help develop the tracking algorithm and qualitatively analyze it. A sample of this dataset was previously shown in Fig. 5.

Afterward, we performed another data collection with a relatively similar setting as in the first, yet with the additional use of a high-speed LiDAR. The LiDAR is placed at the end of the road to provide us with high temporal resolution positional measurements that can be used to estimate the vehicle's position relative to the frame. Benewake TF03-100 high-speed LiDAR was used which has a distance resolution of $1 \mathrm{~cm}$ and an update rate of up to $1000 \mathrm{~Hz}$. A high update rate is essential to collect ground truth data for high-speed applications. This Lidar is accurate up to $\pm 10 \mathrm{~cm}$ (at $10 \mathrm{~m}$ ) and up to $99 \%$ at over $10 \mathrm{~m}$ ( $1 \%$ error). In our case, the Lidar was placed at a range of 60-30 meters of the vehicle, with the vehicle driving towards the Lidar.

For an accurate position estimation, the removal of distortion and the application of perspective transform is crucial to accurately track the objects and estimate their 


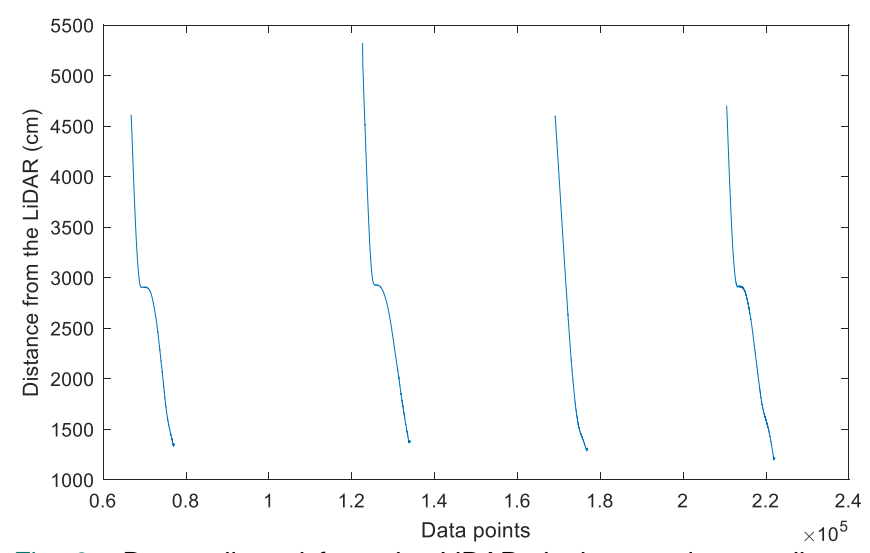

Fig. 8. Data collected from the LiDAR depicts continuous distance measurements of the vehicle being tracked after filtering the data and removing noise. 4 different driving segments were recorded where the vehicle is driving towards the LiDAR until it's no longer in sight. In 3 out of the 4 driving segments, the vehicle that is being tracked decelerates to reach a full stop, then accelerates again. The $3^{\text {rd }}$ driving segment is a continuous non-stop trial.

position. The camera intrinsic matrix is used to remove the distortions from the captured images. This matrix is the result of the camera calibration process. Moreover, 4 points, of known real-life measurements, that correspond to pixels in the frame, are selected in the actual scene and used for the bird's eye perspective transform of the undistorted images. Both of these steps enable proper positional estimation in the form of distance to the LiDAR, which is used to estimate and evaluate the accuracy of our tracking algorithm in traffic monitoring scenes recorded.

The LiDAR, discussed earlier, was used to measure the distance to the vehicle at a sample rate of $1000 \mathrm{~Hz}$ along with a script that reads the serial data over UART at these frequencies, and records timestamps (in nanoseconds) using the system clock for each data point. Then finally, saved to a file to be filtered and post-processed.

As for the post-processing procedure, the data points at which the LiDAR has failed to detect the vehicle are removed. This creates some sporadic missing data points of when the vehicle is being tracked. These missing data points are linearly interpolated to fill in the missing gaps. Finally, a low pass Chebyshev-II filter is used to remove noise and smooth out the positional data. The resulting data is shown in Fig. 8, where several data captures of the vehicle being tracked are demonstrated. Four different driving segments were captured. In three of them $\left(1^{\text {st }}, 2^{\text {nd }}\right.$, and $\left.4^{\text {th }}\right)$, the vehicle decelerates to a full stop in the middle of the camera's frame, before accelerating again in the same direction towards the LiDAR. The other recorded segment shows the vehicle driving continuously without stopping, as represented by the $3 \mathrm{rd}$ driving segment shown in

Fig. 8. A sample of this dataset is shown in Fig. 9.

\section{Results and eVAluation}

Using the dataset discussed in the previous section, some of our hybrid tracking algorithm's results are qualitatively demonstrated in Fig. 9. For the same image frame, we demonstrate the results of different tracking rates compared to the baseline, which is pure frame-based tracking, at a rate of $24 \mathrm{~Hz}$ in our case. Event masks are extracted from the bounding box of the standard image's object detection. We can notice that the density of events decreases as tracking rates increase. This is due to smaller window frame sizes, causing tracking at higher rates to be a bit more challenging if an insufficient amount of events were available.

In these collected samples, the vehicle would be moving sideways only taking into consideration that the road in the scene is oriented horizontally relative to the camera's view. Therefore, quantify the object tracker's performance by estimating its horizontal centroid position given 4 known reallife points (with corresponding distances from each other), and performing perspective transform and camera distortion removal to correct the position of the corresponding center point we're tracking.

Quantitative results are shown in TABLE I. We use different metrics, such as mean absolute and relative errors, as well as root mean squared error (RMSE) for the tracking results of all 4 driving segments shown earlier. We can notice that the error rates do not significantly increase when using higher tracking rates, compared to the base-level performance of the frame-based approach at $24 \mathrm{~Hz}$. This is signified in the mean absolute error and relative error of $11.65 \mathrm{~cm}$ and $0.36 \%$, respectively, of the frame-based tracking, compared to 13.74 $\mathrm{cm}$ and $0.42 \%$ when tracking at $500 \mathrm{~Hz}$ while using YOLO as the frame-based detector. RMSE, similarly, performed very well for high tracking rates in comparison to frame-based tracking. On the other hand, we can notice that the successful tracking rate deteriorates as the tracking rates increase. This is due to the very limited amount of event data generated in 3 of the 4 different driving segments of this dataset when the vehicle decelerates and reaches a complete stop in the middle of the camera's view before it accelerates again leaves the scene. Besides noise, very few event data are generated in a relatively static scene or ones with limited movement. This, combined with the relatively low resolution of the camera used, yields insufficient event data to perform inter-frame tracking, especially at very high rates (using small window frame sizes). However, the successful tracking rate of the continuous segment ( $3^{\text {rd }}$ driving segment shown in Fig. 8) was $100 \%$ for all tracking rates tested. This is due to a sufficient amount of event data that can be used to track the vehicle in the scene at very high tracking rates. Fig. 10 demonstrates these limitations as shown in the plots of the tracking results of two different driving segments compared to the ground truth collected by the LiDAR over time.

It should be noted that one of the main bottlenecks of our approach to initiate object tracking is the requirement of a successful frame-based detection for any new available objects in the scene. Even though in our results, as shown in TABLE I, the frame-based tracking results have a very high successful detection rate of almost $100 \%$ for both object detectors, however, they this isn't always the case. This sets an upper bound for the maximum successful tracking rate our algorithm can reach. However, the reliance on object detectors for the initial frame-based detection can be minimized by only 


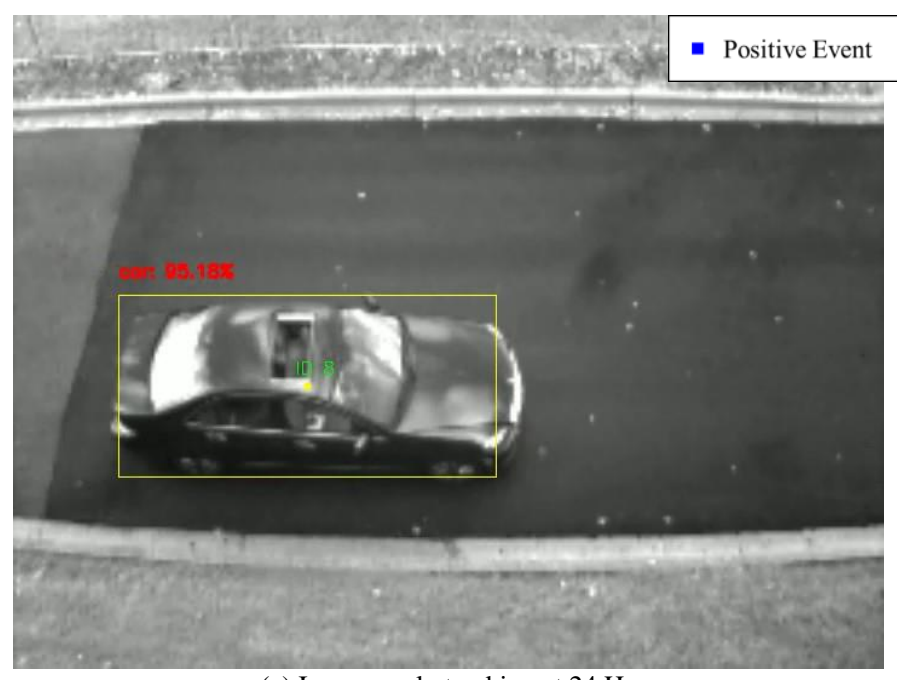

(a) Images-only tracking at $24 \mathrm{~Hz}$

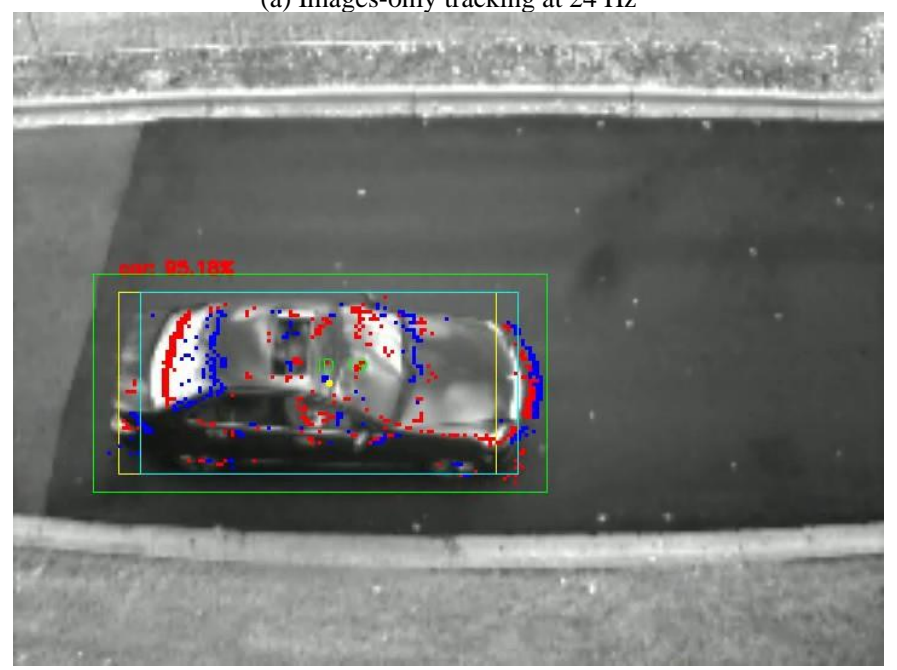

(c) Hybrid tracking at $100 \mathrm{~Hz}$

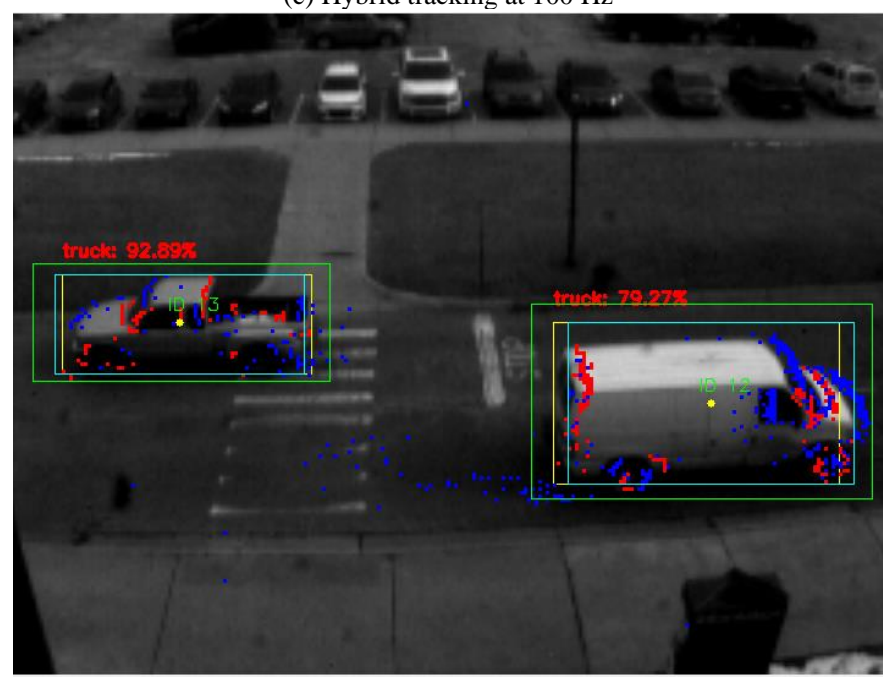

(e) Multi-object tracking is also possible using our Hybrid Tracking algorithm (b) Hybrid tracking at $50 \mathrm{~Hz}$

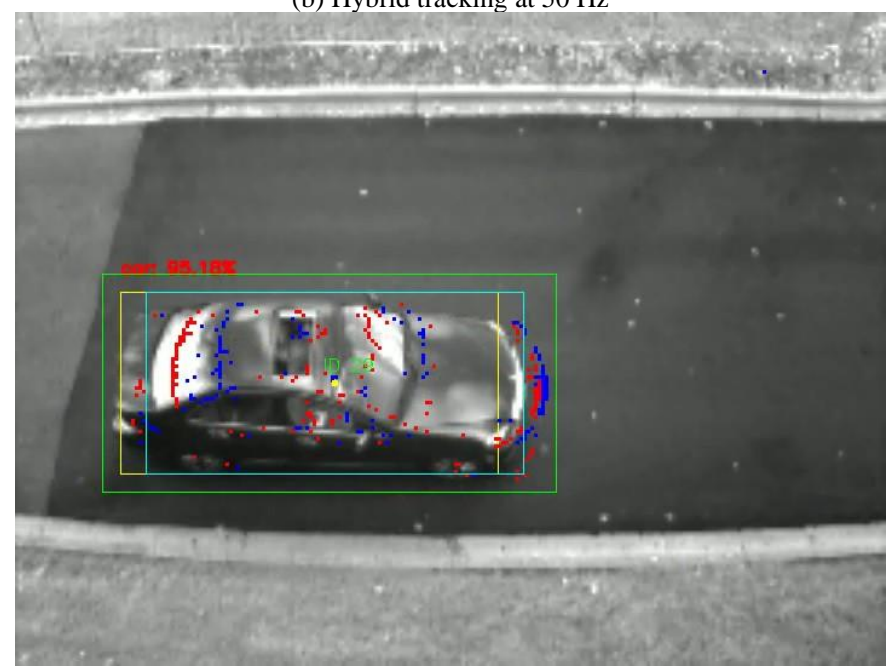

(d) Hybrid tracking at $200 \mathrm{~Hz}$

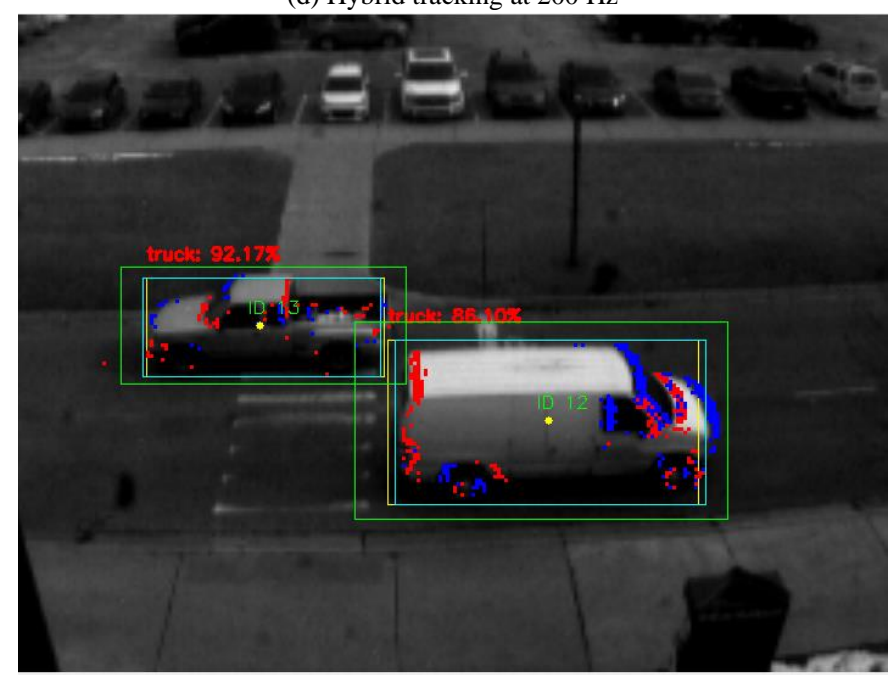

Fig. 9. Qualitative results of our Hybrid Object Tracking algorithm at different rates. (a) shows the tracking using the grayscale image only, whereas the other images show the inter-frame tracking results at different window frame sizes, thus, the different tracking rates are demonstrated by the cyan-colored bounding boxes. Smaller window frame sizes yield higher tracking rates, but fewer events accumulated in each, which can be noticed in the higher amount of events available in (d) compared to (b) and (c). (e) demonstrates the ability to perform multi-object tracking that is robust to partial occlusions. The Yellow bounding box in each represents the initial standard image object detection result. Standard frame object detections demonstrated are generated using YOLOv3. 
TABLE I

HYBRID OBJECT TRACKING ALGORITHM QUANTITATIVE ANALYSIS AT DIFFERENT TRACKING RATES

\begin{tabular}{|c|c|c|c|c|c|c|}
\hline \multirow[b]{2}{*}{ Tracking Rate } & \multicolumn{6}{|c|}{ Metric } \\
\hline & Object Detector & $\begin{array}{l}\text { Mean abs. error } \\
(\mathrm{cm})\end{array}$ & $\begin{array}{c}\text { Mean Relative } \\
\text { Error }\end{array}$ & RMSE & $\begin{array}{l}\text { Successful Tracking } \\
(\%)\end{array}$ & $\begin{array}{l}\text { Successful tracking of the } \\
\text { continuous segment only }(\%)\end{array}$ \\
\hline \multirow{2}{*}{$\begin{array}{c}24 \mathrm{~Hz} \\
\text { (Images only) }\end{array}$} & YOLO & $11.65 \mathrm{~cm}$ & $0.37 \%$ & $15.30 \mathrm{~cm}$ & $99.20 \%$ & $100 \%$ \\
\hline & SSD & $11.56 \mathrm{~cm}$ & $0.36 \%$ & $14.78 \mathrm{~cm}$ & $99.20 \%$ & $100 \%$ \\
\hline \multirow{2}{*}{$50 \mathrm{~Hz}$} & YOLO & $11.69 \mathrm{~cm}$ & $0.37 \%$ & $15.25 \mathrm{~cm}$ & $82.51 \%$ & $100 \%$ \\
\hline & SSD & $12.80 \mathrm{~cm}$ & $0.40 \%$ & $16.28 \mathrm{~cm}$ & $83.27 \%$ & $100 \%$ \\
\hline \multirow{2}{*}{$100 \mathrm{~Hz}$} & YOLO & $12.33 \mathrm{~cm}$ & $0.39 \%$ & $15.93 \mathrm{~cm}$ & $66.24 \%$ & $100 \%$ \\
\hline & SSD & $14.09 \mathrm{~cm}$ & $0.44 \%$ & $17.62 \mathrm{~cm}$ & $68.43 \%$ & $100 \%$ \\
\hline \multirow{2}{*}{$200 \mathrm{~Hz}$} & YOLO & $13.84 \mathrm{~cm}$ & $0.44 \%$ & $17.58 \mathrm{~cm}$ & $50.30 \%$ & $100 \%$ \\
\hline & SSD & $16.25 \mathrm{~cm}$ & $0.5 \%$ & $19.54 \mathrm{~cm}$ & $50.91 \%$ & $100 \%$ \\
\hline \multirow{2}{*}{$500 \mathrm{~Hz}$} & YOLO & $13.74 \mathrm{~cm}$ & $0.42 \%$ & $17.27 \mathrm{~cm}$ & $17.93 \%$ & $100 \%$ \\
\hline & SSD & $20.89 \mathrm{~cm}$ & $0.62 \%$ & $23.8 \mathrm{~cm}$ & $18.77 \%$ & $100 \%$ \\
\hline
\end{tabular}
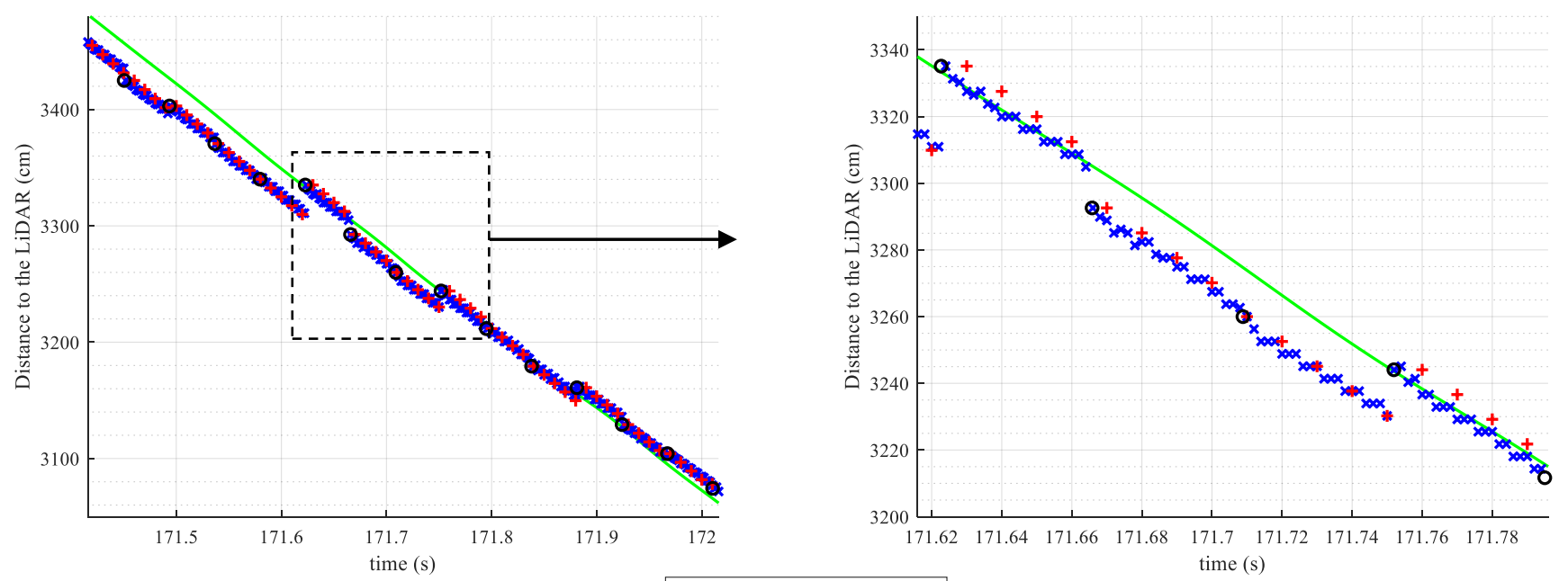

$\begin{array}{ll}\mathbf{x} & \text { Ground truth } \\ \mathbf{+} & \text { 500Hz Hybrid Tracking } \\ \mathbf{0} & \text { 200Hz Hybrid Tracking } \\ & \end{array}$
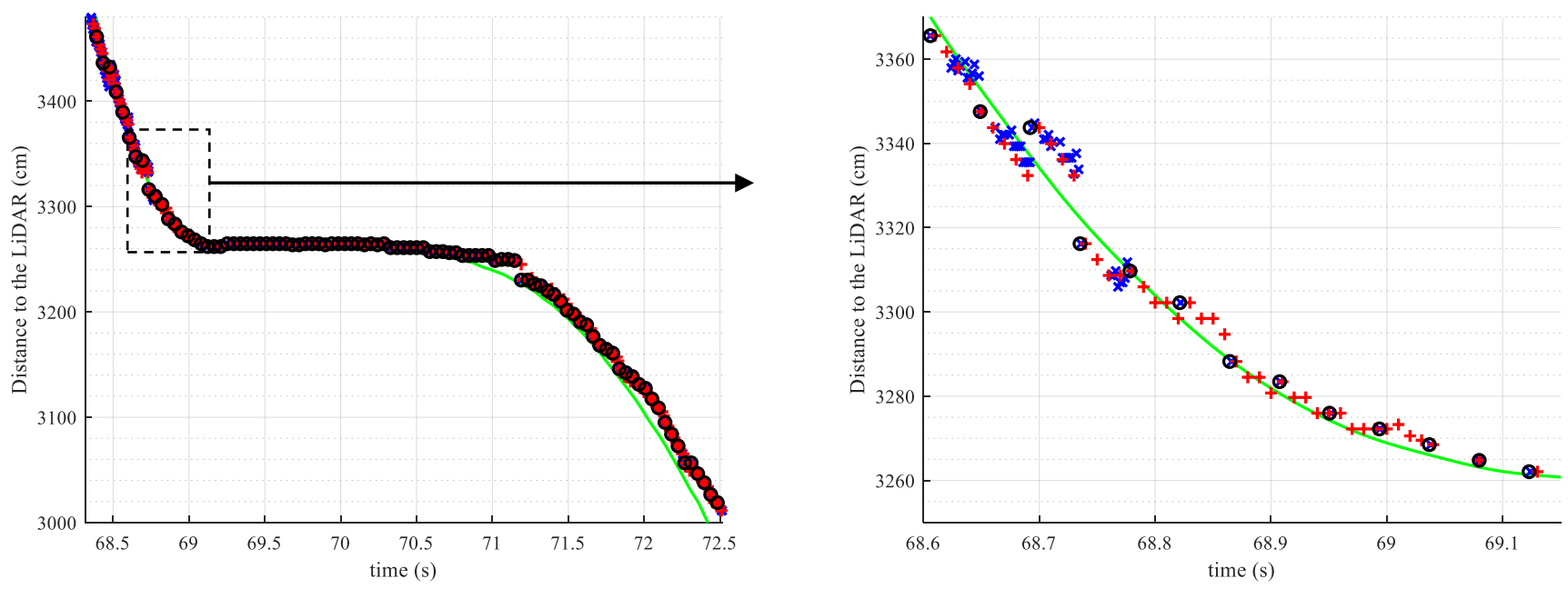

Fig. 10. Scatterplot of the tracking results at different rates vs ground-truth positional data of the LiDAR. The figure demonstrates selected results for two different driving segments (full tracking segment on the left and an enlarged portion on the right). The plots on the top show the results of the continuous driving segment (3rd data segment shown in Fig. 8), and the one on the bottom shows the first driving segment (vehicle decelerates to a full stop before accelerating again). We can see how our approach increases our positional resolution knowledge significantly compared to the baseline frame-based approach. 
requiring the standard image-based detection initially when the object first appears, then fully relying on the continuous stream of event data to resume tracking the objects across the scene while continually updating the event mask corresponding to each object, as discussed previously in our tracking algorithm. Moreover, this hybrid tracking algorithm is designed for fixed cameras with traffic monitoring and tracking as the main application intended. This algorithm works best with objects of fixed and rigid bodies (like vehicles) with few to no moving parts. Further work is required to support objects of different types and shapes with high accuracy.

\section{CONCLUSION}

In this paper, we present our work on the novel, bioinspired, Event cameras. Enabled by their numerous advantages, especially their very high temporal resolution, we present a novel Hybrid Object Tracking algorithm that leverages both standard image frames as well as event data to enable high-speed object tracking. Our algorithm detects

\section{ACKNOWLEDGMENT}

The authors of this paper would like to thank Ella Reimann for her help and assistance in our data collection experiment.

\section{REFERENCES}

[1] C. Papageorgiou and T. Poggio, "A trainable system for object detection," International journal of computer vision, vol. 38, no. 1, pp. 15-33, 2000.

[2] M. Everingham, L. Van Gool, C. K. Williams, J. Winn and A. Zisserman, "The pascal visual object classes (voc) challenge," International journal of computer vision, vol. 88 , no. 2, pp. 303-338, 2010.

[3] P. Gehler and S. Nowozin, "On feature combination for multiclass object classification," in 2009 IEEE 12th International Conference on Computer Vision, 2009.

[4] A. Krizhevsky, I. Sutskever and G. E. Hinton, "ImageNet Classification with Deep Convolutional Neural Networks," in Advances in neural information processing systems, 2012.

[5] J. Long, E. Shelhamer and T. Darrell, "Fully convolutional networks for semantic segmentation," in Proceedings of the IEEE conference on computer vision and pattern recognition, 2015.

[6] B. Leibe, K. Schindler, N. Cornelis and L. Van Gool, "Coupled object detection and tracking from static cameras and moving vehicles," IEEE transactions on pattern analysis and machine intelligence, vol. 30 , no. 10, pp. 1683-1698, 2008.

[7] S. Zhang, C. Wang, S.-C. Chan, X. Wei and C.-H. Ho, "New object detection, tracking, and recognition approaches for video surveillance over camera network," IEEE sensors journal, vol. 15, no. 5, pp. 2679-2691, 2014.

[8] A. Censi and D. Scaramuzza, "Low-latency event-based visual odometry," in 2014 IEEE International Conference on Robotics and Automation (ICRA), 2014.

[9] G. Gallego, T. Delbruck, G. M. Orchard, C. Bartolozzi, B. Taba, A. Censi, S. Leutenegger, A. Davison, J. Conradt, K. Daniilidis and D. Scaramuzza, "Event-based vision: A survey," IEEE Transactions on Pattern Analysis and Machine Intelligence, 2020.

[10] P. Lichtsteiner, C. Posch and T. Delbruck, "A $128 \times 128$ $120 \mathrm{~dB} 15 \mu \mathrm{s}$ Latency Asynchronous Temporal Contrast Vision Sensor.," IEEE journal of solid-state circuits 43.2, pp. 566-576, 2008. objects in the image frames, then utilizes per-object event masks to track objects between consecutive frames. Results show that our tracking algorithm can reach $500 \mathrm{~Hz}$ tracking rates in different scenes, based on a standard image capture rate of $24 \mathrm{~Hz}$. To quantify and analyze the performance of our tracking algorithm, we set up a data collection experiment using a fixed DAVIS camera to track moving vehicles across the scene, along with a LiDAR to estimate the vehicle's horizontal position to compare that with the positional output of our algorithm. Results show relatively high tracking accuracy at very high update rates (up to $500 \mathrm{~Hz}$ ) with vehicles of well-defined edges, which leads to clear event data being generated. The main challenge was the low resolution of the camera used, imposing a bottleneck of the position and tracking accuracy of objects moving at very low speeds. This algorithm performs optimally when tracking high-speed moving objects of somewhat fixed shapes that generate clear and sufficient event data that overcomes events generated due to noise.

[11] T. Bolten, R. Pohle-Frohlich and K. D. Tonnies, "DVSOUTLAB: A Neuromorphic Event-Based Long Time Monitoring Dataset for Real-World Outdoor Scenarios," in Proceedings of the IEEE/CVF Conference on Computer Vision and Pattern Recognition, 2021.

[12] D. Falanga, K. Kleber and D. Scaramuzza, "Dynamic obstacle avoidance for quadrotors with event cameras," Science Robotics, vol. 5, no. 40, 2020.

[13] D. Falanga, S. Kim and D. Scaramuzza, "How fast is too fast? the role of perception latency in high-speed sense and avoid," IEEE Robotics and Automation Letters, vol. 4, no. 2, pp. 1884-1891, 2019.

[14] L. Pan, C. Scheerlinck, X. Yu, R. Hartley, M. Liu and Y. Dai, "Bringing a blurry frame alive at high frame-rate with an event camera," in Proceedings of the IEEE/CVF Conference on Computer Vision and Pattern Recognition, 2019.

[15] D. Gehrig, M. Gehrig, J. Hidalgo-Carrió and D. Scaramuzza, "Video to events: Recycling video datasets for event cameras $\}$," in Proceedings of the IEEE/CVF Conference on Computer Vision and Pattern Recognition, 2020.

[16] H. Rebecq, R. Ranftl, V. Koltun and D. Scaramuzza, "Events-to-video: Bringing modern computer vision to event cameras," in Proceedings of the IEEE/CVF Conference on Computer Vision and Pattern Recognition, 2019.

[17] M. Litzenberger, C. Posch, D. Bauer, A. N. Belbachir, P. Schon, B. Kohn and H. Garn, "Embedded vision system for real-time object tracking using an asynchronous transient vision sensor," in 2006 IEEE 12th Digital Signal Processing Workshop \& 4th IEEE Signal Processing Education Workshop, 2006.

[18] D. Tedaldi, G. Gallego, E. Mueggler and D. Scaramuzza, "Feature detection and tracking with the dynamic and active-pixel vision sensor (DAVIS)," in 2016 Second International Conference on Event-based Control, Communication, and Signal Processing (EBCCSP), 2016.

[19] D. Gehrig, H. Rebecq, G. Gallego and D. Scaramuzza, "EKLT: Asynchronous, Photometric Feature Tracking using Events and Frames," International Journal of Computer Vision, 2019.

[20] J. Kim, J. Bae, G. Park, D. Zhang and Y. M. Kim, "NImageNet: Towards Robust, Fine-Grained Object Recognition With Event Cameras," in Proceedings of the IEEE/CVF International Conference on Computer Vision, 2021.

[21] A. Sironi, M. Brambilla, N. Bourdis, X. Lagorce and R. 
Benosman, "HATS: Histograms of averaged time surfaces for robust event-based object classification," in Proceedings of the IEEE Conference on Computer Vision and Pattern Recognition, 2018.

[22] A. Yilmaz, O. Javed and M. Shah, "Object tracking: A survey," Acm computing surveys (CSUR), vol. 38, no. 4, pp. 12-es, 2006.

[23] B. Deori and D. M. Thounaojam, "A survey on moving object tracking in video," International Journal on Information Theory (IJIT), vol. 3, no. 3, pp. 31-46, 2014.

[24] J. Redmon and A. Farhadi, "YOLOv3: An Incremental Improvement," arXiv, 2018.

[25] W. Liu, D. Anguelov, D. Erhan, C. Szegedy, S. Reed, C.-Y. Fu and A. C. Berg, "SSD: Single Shot MultiBox Detector," in European conference on computer vision, 2016.

[26] P.-E. Danielsson, "Euclidean distance mapping," Computer Graphics and image processing, vol. 14, no. 3, pp. 227-248, 1980.

[27] A. Rosebrock, "Simple Object Tracking with OpenCV," PyImageSearch, [Online]. Available: https://www.pyimagesearch.com/2018/07/23/simple-objecttracking-with-opencv/. [Accessed 110 2021].

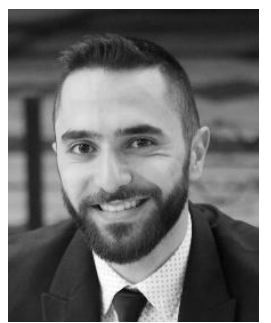

Zaid A. El Shair received the B.Sc. degree in computer engineering from Princess Sumaya University for Technology, Amman, Jordan, in 2018 and the M.S.E. degree in Computer Engineering from the University of MichiganDearborn, Ml, in 2019. He is currently a Ph.D. candidate in Electrical, Electronics, and Computer Engineering, at the University of Michigan-Dearborn, working within the domain of Computer Vision. Recently, he has been researching and developing methods for robust high-speed object tracking using the very recent, bio-inspired, Event Cameras. Research experience includes Networked-Embedded Systems, Machine Learning, Intelligent systems, and Computer Vision.

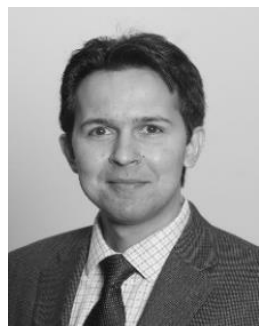

Samir A. Rawashdeh (M'05, SM'20) received the B.S. degree in electrical engineering from the University of Jordan, Amman, Jordan in 2007, and the M.S.E.E. and Ph.D. degrees in 2009 and 2013 in electrical engineering from the University of Kentucky, Lexington, KY. He is currently an Associate Professor in the Electrical and Computer Engineering department at the University of Michigan Dearborn, which he joined in 2014. His research activities and interests include robot perception, embedded systems, and smart health. 